\title{
ANATOMICAL VARIATIONS AND DISTRIBUTIONS OF OBTURATOR NERVE ON ETHIOPIAN CADAVERS
}

Berhanu KA, Taye M, Abraha M, Girma A

Correspondence to Berhanu Kindu Ashagrie Email: adaneberhanu23@gmail.com; Tele: +251966751721; PO Box: 272 Debre Tabor University , North Central Ethiopia

\begin{abstract}
Variations in anatomy of the obturator nerve are important to surgeons and anesthesiologists performing surgical procedures in the pelvic cavity, medial thigh and groin regions. They are also helpful for radiologists who interpret computerized imaging and anesthesiologists who perform local anesthesia. This study aimed to describe the anatomical variations and distribution of obturator nerve. The cadavers were examined bilaterally for origin to its final distribution and the variations and normal features of obturator nerve. Sixty-seven limbs sides (34 right and 33 left sides) were studied for variation in origin and distribution of obturator nerve. From which $88.1 \%$ arises from L2, L3 and L4 and; $11.9 \%$ from L3 and L4 spinal nerves. In $23.9 \%, 44.8 \%$ and $31.3 \%$ of specimens the bifurcation levels of obturator nerve were determined to be intrapelvic, within the obturator canal and extrapelvic, respectively. The anterior branch subdivided into two, three and four subdivisions in $9 \%, 65.7 \%$ and $25.4 \%$ of the specimens, respectively, while the posterior branch provided two subdivisions in $65.7 \%$ and three subdivisions in $34.3 \%$ of the specimens. Hip articular branch arose from common obturator nerve in $67.2 \%$ to provide sensory innervation to the hip joint. Accessory obturator nerve was not observed at all in this study.

Key words: Variations, obturator nerve
\end{abstract}

\section{INTRODUCTION}

Obturator nerve originates from the lumbar plexus. The lumbar plexus is formed by the first three and most of the fourth lumbar ventral rami, with a contribution from the 12th thoracic ventral ramus (Standring et al. 2008). Commonly obturator nerve arises from ventral divisions of the second, third, and fourth lumbar roots of the lumbar plexus. However, variations are found with regard to the absence of the second spinal root in its formation (Horwitz 1939; Arora et al. 2014). Accessory obturator nerve is occasionally present in $10 \%$ of population. It forms part of the lumbar plexus commonly arising from ventral branches of the third and fourth lumbar ventral rami (Standring et al. 2008).

Obturator nerve block is not routinely performed because nerve localization and clinical evaluation of the nerve block's success is difficult and time consuming. One possible reason for these difficulties is the high anatomic variability of the nerve in the inguinal region. Studies have described anatomic variations of obturator nerve, but less is known about the exact number of divisions and subdivisions in the inguinal region. Complications related to damage to the obturator nerve and its branches intraoperatively and postoperatively have been reported in different literatures (Bradshaw et al. 1997, Possever et al. 1998, Loeb et al. 2010, Ates et.al 2013, Ball et al. 2013). Damage to the obturator nerve during surgical and clinical procedures may occur if knowledge of the variation in the branching patterns of the nerve is lacking or incorrect. Damage to obturator nerve presents with motor and sensory losses such as paraesthesia of the medial third of the thigh and weakness of 
the adductor muscles (Benes 1999, Spaliveiro et al. 2004, Ghaemmaghami et al. 2009). Symptoms of obturator nerve damage have been observed in pelvic surgery such as radical prostatectomy, cystectomy, pelvic lymphadenectomy and procedures for treatment of stress urinary incontinence (Spaliveiro et al., 2004; Hazewinkel et al., 2009; Kitagawa et al., 2009, Shah et al., 2013). With regard to regional anesthesia; obturator nerve block is commonly performed to prevent sudden thigh adduction during transurethral resection of bladder tumor, to provide optimal analgesia for knee surgery, to treat chronic hip pain and to improve persistent hip adductor spasticity (Yoshida, 2017). Anesthesia of the obturator nerve and its branches failed blocks or even nerve damage have been reported when an obturator nerve block for knee and hip surgery is incorrectly performed (Bouaziz et al., 2002; Chequet et al. 2005; Simeoforidou et al. 2013).

These problems further emphasize the importance of clear description of variations of the obturator nerve. This study provides a detailed description of the variability in the origin, level of bifurcation into anterior and posterior branches and the distribution of the obturator nerve in a series of cadavers. It is helpful in the implementation of regional anesthesia, pelvic surgical procedures and understanding of possible variations for Anatomists.

The aim of this study was assessment of anatomical variations and distribution of obturator nerve on 34 Ethiopian cadavers in selected medical schools in Ethiopia.

\section{METHOD S AND MATERIALS}

Embalmed human specimens in dissection rooms of Anatomy departments of selected public universities in Ethiopia (University of Gondar, St. Paul's Millennium Medical College, Bahir Dar, Addis Ababa, Jimma, Debre Markos, and Debre Tabor Universities) were used.

Obturator nerve was examined bilaterally from the origin to its final distribution and the variations and normal features were recorded using checklist and photographed. The specimens were carefully examined and inspected with naked eye in detail; root values for origin, levels of bifurcation of its anterior and posterior branches, the distribution of subdivisions to individual muscles, the branch to hip articular joint and presence or absence of accessory obturator nerve on well dissected and cleaned cadavers. For more detailed description, after removal of the skin and the subcutaneous tissues of the posterior abdominal wall and greater pelvis the nerve was exposed to observe variations of origin and bifurcation. In the anterior and medial thigh, the sartorius, pectineus, and adductor longus muscles were dissected free from the deep fascias. With great care, the pectineus and adductor longus muscles were dissected at their fascial septum and were incised in a transverse pattern to expose the adductor brevis and external obturator muscles lying at a deeper level. The anterior branch of the obturator nerve was identified within the fascia covering adductor brevis muscle. The course of the posterior branch of the obturator nerve was exposed after dissection and anterolateral reflection of the adductor brevis muscle. Both branches of the obturator nerve were followed cranially and caudally, and they were carefully dissected from the surrounding tissues. Special attention was given to the point where the common obturator nerve divided into its anterior and posterior branches, as well as to the identification of the hip articular branch. After careful observation the data was recorded in the properly designed checklist and the nerve was photographed using camera and presented on the result. 


\section{RESULTS}

Out of 67 sides $88.1 \%$ (59 sides) had origin of obturator nerve from L2, L3 and L4 lumbar spinal nerves and $11.9 \%$ (8 sides) originate only from L3 and L4 lumbar spinal nerves.

In $23.9 \%$ (16 sides) of specimens the division was intrapelvic. The division was located within the obturator canal in $44.8 \%$ (30 sides) of cases. In $31.3 \%$ ( 21 sides) of specimens, the nerve bifurcated into anterior and posterior branches in the extrapelvic region. In the subdivisions of anterior branch; three muscular subdivisions were observed in $65.7 \%$ (44 sides) of the specimens. Two muscular divisions and four muscular divisions were also observed as shown in table 1 . In the subdivision of posterior branch; $65.7 \%$ of the specimens had two subdivisions from the posterior branch of the obturator nerve which provide innervation to the obturator externus and adductor magnus muscles. In $34.3 \%$ of the specimens three muscular divisions were distributed to the adductor magnus, obturator externus and adductor brevis muscles. The adductor brevis muscle was observed having a double innervation pattern by both the anterior and posterior branches of obturator nerve. The observation of the hip articular branch of the obturator nerve shows $67.2 \%$ of the specimens had hip articular branch originated from the common obturator nerve (as shown in Figure 4a). It also originates from anterior and posterior branches as presented in table 2 . In this study, accessory obturator nerve was not observed in any of the cadavers in the 67 samples of the study.
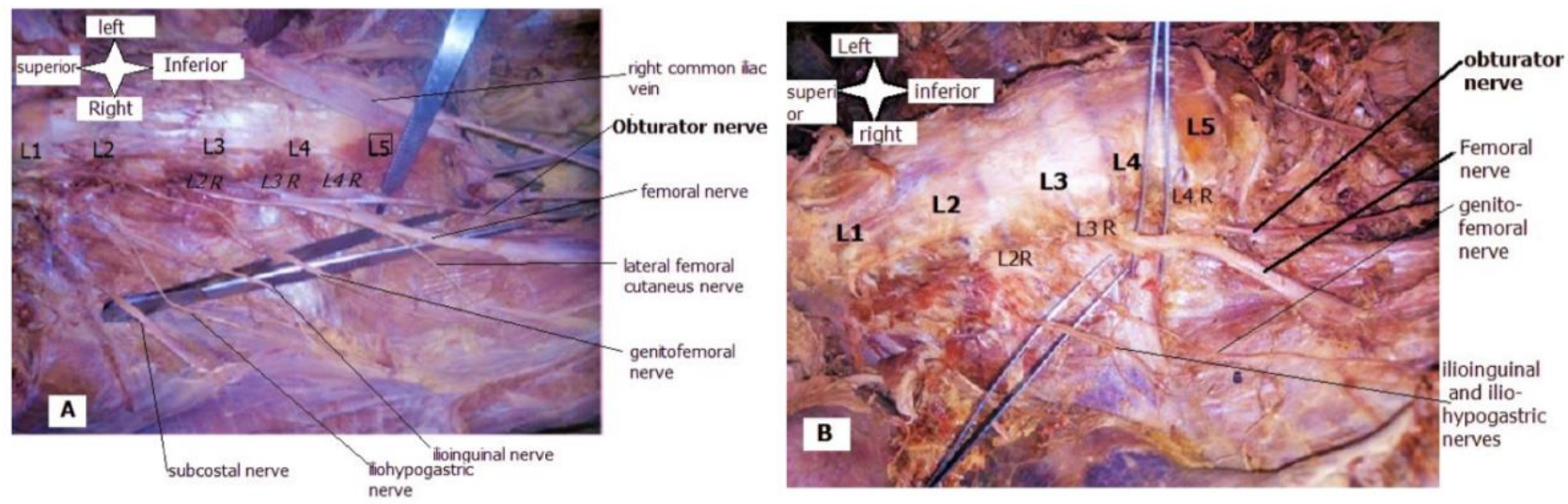

Figure1: Photographs showing the lumbar plexus and origins of obturator nerve, a cadaveric study in Ethiopia, 2018 ( $\mathrm{n}$ =67). The photographs show the right lumbar plexuses of different human specimen. In ' $A$ ' it is shown that the origin of obturator nerve is from L2, L3 and L4 anterior rami lumbar spinal nerves and in ' $\mathrm{B}$ ' the origin of obturator nerve is from only $\mathrm{L} 3$ and $\mathrm{L} 4$ anterior rami lumbar spinal nerves. Where: $\mathrm{L} 2 \mathrm{R}=$ lumbar 2 root (has no contribution in B), L3R = lumbar 3 root, $\mathrm{L} 4 \mathrm{R}=$ lumbar 4 root.
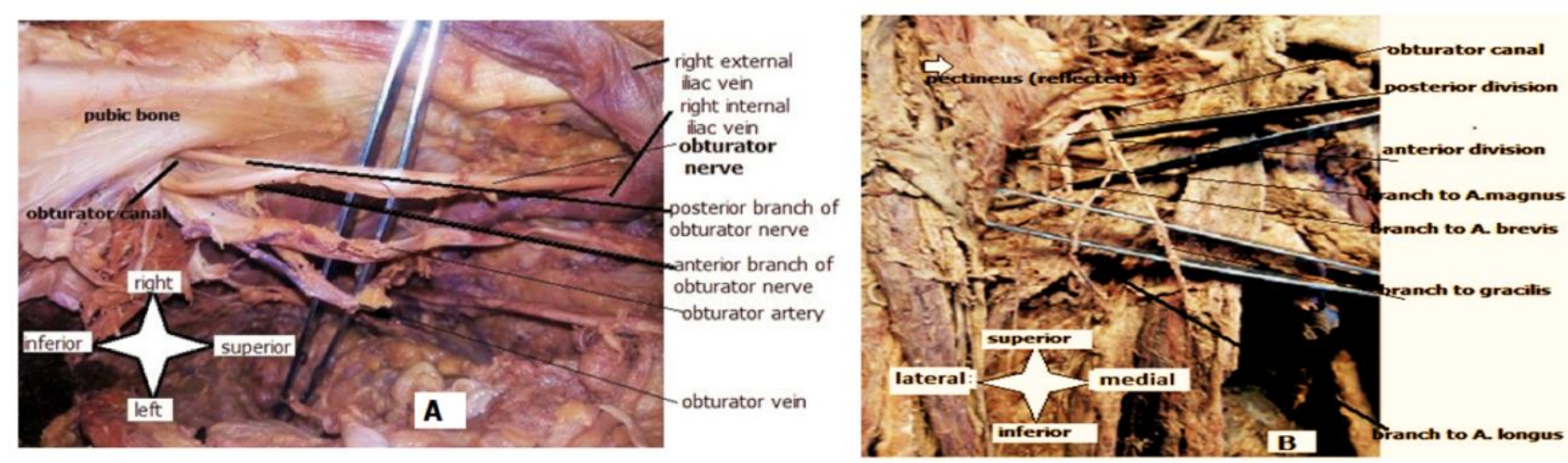

Figure 2: Photographs of right sides of human specimen showing the bifurcation level and distribution of obturator nerve, a cadaveric study in Ethiopia, $2018(\mathrm{n}=67)$. A) Intrapelvic bifurcation B) extrapelvic bifurcation of obturator nerve and the anterior branch subdivided into two branches: to gracilis and adductor longus muscles. 
Table 1: The percentage frequency of subdivisions from the anterior branch of obturator nerve, a cadaveric study in Ethiopia, 2018 ( $n=67)$.

\section{Number of subdivisions of anterior branch}

2 branches: to the $A$. longus and gracilis Percentage (\%)

3 branches: to $A$. longus, $A$. brevis and gracilis 9

4 branches: to $A$. longus, A. brevis, gracilis and pectineus

25.4

Key: $\mathrm{A}=$ Adductor

Table 2: Variations in the origin of hip articular nerve from obturator nerve, a cadaveric study in Ethiopia, 2018 ( $n=67)$

\begin{tabular}{|r|c|}
\hline Origin of hip articular branch & Percentage $(\%)(n=67)$ \\
\hline $1 . \quad$ Common obturator nerve & 67.2 \\
\hline 2. Anterior obturator nerve branch & 17.9 \\
\hline $3 . \quad$ Posterior obturator nerve branch & 14.9 \\
\hline
\end{tabular}

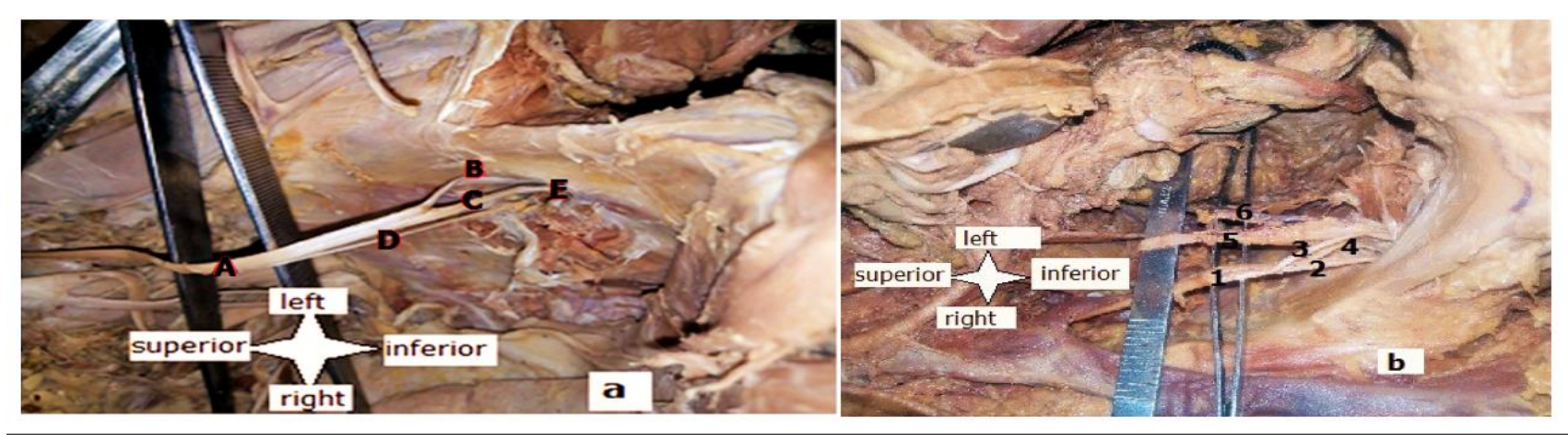

Figure 3: Photographs of specimens showing the origin of hip articular branch of obturator nerve, cadaveric study in Ethiopia, 2018 ( $n=67)$. Photograph 'a'(left side) shows origin of hip articular branch from common obturator nerve; where $\boldsymbol{A}$ : common obturator nerve, $\boldsymbol{B}$ : anterior division of obturator nerve, $\boldsymbol{C}$. posterior division of obturator nerve, $\boldsymbol{D}$ : hip articular branch and $\boldsymbol{E}$; obturator canal. Photograph 'b'(right side) shows origin of hip articular branch from posterior division of obturator nerve; where 1: common obturator nerve, 2: posterior division of obturator nerve 3: anterior division of obturator nerve 4: hip articular branch arose from posterior division, 5: obturator artery and 6: obturator vein.

\section{DISCUSSION}

Adequate knowledge of the anatomy of the obturator nerve and its branches is quite helpful in the application of clinical and surgical procedures such as locating and blocking the nerve. The results of the present anatomic study clearly demonstrated that the presence of variations in origin, branching pattern and distribution of the obturator nerve.

In the present study majority $(88.1 \%)$ of obturator nerve originated from L2, L3 and L4 lumbar spinal roots. This is supported by study done in South Africa with 181 sides which was 80\% (Tshabala 2015) and study done in USA with 38 sides which was $100 \%$ (Anloague and Huijbregts 2009). Other studies done in USA
(Horwitz 1939) and India (Arora et al. 2014) observed lower prevalence from this origin which was $10 \%$ and $33 \%$, respectively. In addition, the later studies identified that obturator nerve has also origin from spinal roots $\mathrm{T} 12-\mathrm{L} 5$ but in this study this finding was not observed. This clearly shows presence of variability in the origin of obturator nerve. Knowing the presence of variation in the origin of obturator nerve is important for anesthetists for effective blocking of the nerve when surgical procedures are performed at the hip joint, proximal part of medial thigh or knee joint, where the nerve provides sensory innervation. 
Obturator nerve bifurcates into anterior and posterior branches as well as branch to hip articular joint during its course around the obturator canal. $23.9 \%$ of the specimens having intrapelvic bifurcation of obturator nerve into anterior and posterior branches is similar with a study conducted in Greece with 168 sides which was $23.22 \%$ (Anagnostopoulu et al. 2009) and high when compared with the study done in South Africa (Tshabalala 2015) with 181 sides which was $2 \%$ intrapelvic. On the other hand, $44.8 \%$ of the specimens have bifurcation within the obturator canal in which this finding is nearer to the study done in Greece which was $51.78 \%$ (Anagnostopoulu et al.2009) and is lower when compared with the South African report which was 93\% (Tshabalala 2015) for bifurcation of obturator nerve within the obturator canal. Furthermore, $31.3 \%$ of the specimens have extrapelvic bifurcation of obturator nerve which is approaching to the finding of the study done in Greece which was $25 \%$ and has higher percentage than the study in South Africa which was $5 \%$ extrapelvic.

The present study had closer findings to the study conducted in Greece and clearly different finding to the study done in South Africa. This shows that the bifurcation level of obturator nerve is variable which in turn concern localization of the nerve in the clinical and surgical procedures. This high variability of bifurcation makes difficulty in blocking and locating selectively the anterior or posterior branches during pelvic, hip joint and medial thigh clinical procedures. Additional literatures couldn't be found that investigate the bifurcation patterns of the obturator nerve around the obturator canal.

In the present study $9 \%, 65.7 \%$ and $25.4 \%$ of the specimens have two, three and four subdivisions, respectively. This observation was approaching to a similar study conducted in Greece with 168 sides demonstrated that $28.57 \%$ have two subdivisions, $66.66 \%$ have three subdivisions and $4.76 \%$ have four subdivisions (Anagnostopoulu et al. 2009). These observations have been also documented by another researcher in South Africa (Tshabalala 2015), which had observed the subdivisions of anterior obturator nerve to the medial thigh muscles were variable.

There was also variation of subdivisions in the posterior branch; where $65.7 \%$ of the specimens have two subdivisions, $34.3 \%$ have three subdivisions; but one and four subdivisions from posterior branch of obturator nerve were not observed in this study.

Table 3: Comparison of various studies for variations in origin of the obturator nerve, cadaveric study in Ethiopia, $2018(n=67)$

\begin{tabular}{|l|l|l|l|l|l|}
\hline Studies & Country & $\begin{array}{l}\text { Sample } \\
\text { (n) }\end{array}$ & \multicolumn{3}{|c|}{ Prevalence of origin (\%) } \\
\cline { 4 - 6 } & & L2 - L4 & L3 \&L4 & T12 - L5 \\
\hline Horwitz 1939 & USA & 228 & 10 & 77 & 13 \\
\hline Anloague and Huijbregts 2009 & USA & 38 & 100 & 0 & 0 \\
\hline Arora et al. 2014 & India & 60 & 33 & 65 & 2 \\
\hline Tshabalala 2015 & South Africa & 181 & 80 & 20 & 0 \\
\hline Current study 2018 & Ethiopia & 67 & 88.1 & 11.9 & 0 \\
\hline
\end{tabular}

Table 4: Comparison of prevalence of accessory obturator nerve in various studies, cadaveric study in Ethiopia, $2018(n=67)$.

\begin{tabular}{|l|c|c|c|}
\hline \multicolumn{1}{|c|}{ Studies } & Country & Sample $(\mathrm{n})$ & Prevalence (\%) \\
\hline Tubbs et al. 2005 & USA & 22 & 0 \\
\hline Akkaya et al. 2008 & Turkey & 24 & 12.5 \\
\hline Anloague and Huijbregts 2009 & USA & 38 & 8.8 \\
\hline Tshabalala 2015 & South Africa & 181 & 0 \\
\hline Archana et.al 2016 & India & 50 & $8 \%$ \\
\hline Current study 2018 & Ethiopia & 67 & 0 \\
\hline
\end{tabular}


Similar study conducted in Greece with 168 sides demonstrated that $13.69 \%$ have one subdivision, $60.11 \%$ have two subdivisions, $19.04 \%$ have three subdivisions and $7.14 \%$ have four subdivisions (Anagnostopoulu et al. 2009). The two subdivisions were most frequent in both studies, which show presence of clear variations in the subdivision pattern with the most frequent two smaller branches. The anatomical variations are important to consider blocking of the posterior branch of the obturator nerve with a local anesthesia to assist in post-operative analgesia of patients that have undergone knee surgery in combination with other blocks.

The hip articular branch of obturator nerve is a sensory branch to the hip joint. This nerve may arise from the common obturator nerve, from the anterior branch of obturator nerve or from the posterior branch of obturator nerve. However, the most common was described to be from the common obturator nerve (Anagnostopoulu et al. 2009). In this study the majority $(67.2 \%)$ of it originate from common obturator nerve. This study also strengthens the finding of the study in Greece in which the most frequent origin of hip articular branch is from common obturator nerve.
As described earlier, accessory obturator nerve was not observed in this particular study. This failure of occurrence of the accessory obturator nerve might be due to small size of the sample, variation of the nerve from population to population or its rare prevalence in the general population which was described to be about $10 \%$ (Standring et al. 2008). However, different researchers found different prevalence of the nerve in their observation, as explained in the literature. Therefore, considering the presence of this nerve during hip surgery is important, since it supplies sensory to hip joint when present.

In conclusion, there are noteworthy variations with regard to the prevalence of variations of the root values of the obturator nerve in the lumbar area; the level of bifurcation of the obturator nerve into anterior and posterior branches around the obturator canal and the distribution of the anterior and posterior branches of the obturator nerve in the medial compartment of the thigh. Teachers of anatomy and clinicians should be wary of these variations.

\section{REFERENCES}

1. Akkaya T, Comert A, Kendir S, Acar H, Gumus H, Tekdemir I, Elhan A. 2008. Detailed anatomy of accessory obturator nerve blockade. Minerva Anestesiol. 74: 119-122

2. Anloague PA, Huijbregts P. 2009. Anatomical variations of the lumbar plexus: a descriptive anatomical study with proposed clinical implications. J. Man. Manip. Ther. 17:107-114.

3. Archana BJ, Nagaraj DN, Pradeep P, Lakshmi PS. 2016. Anatomical variations of accessory obturator nerve: a cadaveric study with proposed clinical implications. IJAR. 4: 2158-61.

4. Arora D, Kaushal S, Singh G. 2014. Variations of lumbar plexus in 30 adult human cadavers; a unilateral prefixed plexus. IJPAES. 4(4): $225-228$.

5. Ates S, Tulandi T. 2013. Malpractice claims and avoidance of complications in endoscopic surgery. Best Pract Res Clin Obstet Gynecol. 27: 349-361.

6. Ball MW, Gorin MA, Allaf M. E. 2013. Reducing morbidity of pelvic and retroperitoneal lymphadenectomy. Curr Urol Rep. 14: 488-495.

7. Bouaziz H, Vial F, Jochum D, Macalou D, Heck M, Meuret P, Braun M, Laxenaire MC. 2002. An evaluation of the cutaneous distribution after obturator nerve block. Anesth Analg. 94: 445449.

8. Bradshaw C, Mccrory P, Bell S, Brukner P. 1997. Obturator Nerve Entrapment A Cause of Groin Pain in Athletes. Am. J Sports Med. 25: 402-408.

9. Ghaemmaghami F, Behnamfar F, Saberi H. 2009. Immediate grafting of transected obturator nerve during radical hysterectomy. IJS. 7:168-169. 
10. Hazewinkel M. H., Hinoul P. and Roovers J. P. 2009. Persistent groin pain following a transobturator sling procedure for stress urinary incontinence: a diagnostic and therapeutic challenge. Int Urogynecol J. 20:363-365.

11. Horwitz MT. 1939. The anatomy of (a) the lumbosacral nerve plexus - its relation to variations of vertebral segmentation, and (b), the posterior sacral nerve plexus. Anat Rec. 74: $91-107$.

12. Kitagawa R, Kim D, Reid N, Kline D. 2009. Surgical management of obturator nerve lesions. NEUROSURGERY. 65:24-28.

13. Loeb S, Partin AW, Schaeffer EM. 2010. Complications of pelvic lymphadenectomy: do the risks outweigh the benefits? Nat Rev Urol. 12: 20.

14. Shah DJ, Andi AC, Ramesar K, Gillian Watson M. 2013. Adductor muscle atrophy due to obturator nerve compression by metastatic lymph node enlargement-A rare complication of recurrent bladder cancer. OMICS J Radiol. DOI: 10.4172/2167-7964.1000122.

15. Simeoforidou M, Bareka M, Basdekis G, Tsiaka K, Chantzi E, Vretzakis G. 2013. Peripheral nerve blockade as an exclusive approach to obturator nerve block in anterior cruciate ligament reconstructive surgery. Korean J Anesthesiol. 65: 410-417.

16. Standring S. 2008. The pelvic girdle and lower limb. Gray's Anatomy; the anatomical basis of clinical practice. 40th ed. London Churchill Livingstone; 76- 77.

17. Tshabalala ZN. 2015. The anatomy and clinical implications of the obturator nerve and its branches. University of Pretoria - e repository. http://hdl.handle.net/2263/53048

18. Tubbs RS, Salter EG, Wellons Iii JC, Blount JP, Oakes WJ. 2005. Anatomical landmarks for the lumbar plexus on the posterior abdominal wall. J Neurosurg. 2: 335-338

19. Yoshida T. 2017. Ultrasound-Guided Obturator Nerve Block: A Focused Review on Anatomy and Updated Techniques. BioMed Res. doi.org/10.1155/2017/7023750 